\title{
Decreased estimated glomerular filtration rate predicts long-term recurrence after catheter ablation of atrial fibrillation in mild to moderate renal insufficiency
}

\author{
Jing Zheng ${ }^{1}$, Deling Zu ${ }^{1}$, Keyun Cheng ${ }^{1}$, Yunlong Xia ${ }^{2}$, Yingxue Dong ${ }^{2 *}$ and Zhenyan Gao ${ }^{{ }^{*}}$
}

\begin{abstract}
Background: Catheter ablation is an established therapy for atrial fibrillation (AF), but recurrence after ablation remains a great challenge. Additionally, little is known about the effect of renal function on the efficiency of AF ablation. This study aimed to evaluate the predictors of the prognosis of catheter ablation for AF, especially the effect of renal function.

Methods: A total of 306 drug-refractory symptomatic patients with AF who underwent first-time catheter ablation were enrolled in the present study. Individuals underwent circumferential pulmonary vein isolation for paroxysmal AF and stepwise ablation for persistent AF.

Results: The follow-up time was $27.2 \pm 19.5$ months, 202 patients (66.01\%) were free of atrial tachyarrhythmia (nonrecurrence group), and the other 104 patients experienced recurrence (recurrence group). The recurrence group had a larger left atrial diameter (LAD) and left atrial volume (LAV), a higher $L A V$ index (LAVI) (both, $p<0.01)$, and a lower estimated glomerular filtration rate (eGFR) $\left(53.5 \pm 14.4 \mathrm{vs} .65 .5 \pm 13.3 \mathrm{ml} / \mathrm{min} / 1.73^{2}, p<0.001\right)$ and creatinine clearance rate (CCr) $(85.2 \pm 26.1$ vs. $101.5 \pm 29.4 \mathrm{ml} / \mathrm{min}, p<0.05)$. Multivariate logistic regression indicated both eGFR $(p=0.002)$ and LAVI $(p<0.001)$ as independent associated factors for long-term recurrence after single catheter ablation; multivariate Cox proportional hazard regression with backward feature selection identified both eGFR (HR: 0.93, 95\% Cl: $0.91-0.95, p<0.001)$ and LAVI (HR: 1.32, 95\% Cl: 1.25-1.40, $p<0.001)$ as independent prognostic factors for recurrence when adjusting other clinical variables.
\end{abstract}

Conclusions: Decreased eGFR and elevated LAVI may facilitate the long-term recurrence of atrial tachyarrhythmia after catheter ablation for AF.

Keywords: Estimated glomerular filtration rate, Atrial fibrillation, Catheter ablation, Recurrence, Mild to moderate renal insufficiency

*Correspondence: dlsusan@126.com; gaozhenyan80@163.com

${ }^{1}$ Department of Cardiology, The Quzhou Affiliated Hospital of Wenzhou Medical University, Quzhou People's Hospital, Quzhou 324000, Zhejiang, China

${ }^{2}$ Department of Cardiology, The First Affiliated Hospital of Dalian Medical University, Dalian 116000, Liaoning, China

\section{Background}

Atrial fibrillation (AF) is a common arrhythmia that accounts for a significantly increased risk of stroke and all-cause mortality [1-3]. Catheter ablation has evolved over the past decade and has been demonstrated as an established therapy for paroxysmal AF and persistent AF [4]. However, recurrence after ablation is still a great 
challenge. In clinical work, standardized and reasonable evaluation of ablation prognosis is beneficial to optimize the selection of individualized treatment and reduce additional medical expenditure. There are several identical risk factors between AF and renal insufficiency, such as hypertension, diabetes mellitus, and age [5]. Additionally, a higher prevalence of AF has been reported among patients with different stages of chronic renal disease [6]. However, the effect of renal function on the efficiency of AF ablation has not been well elucidated. The present study aimed to retrospectively analyse the risk factors for recurrence after first-time catheter ablation of $\mathrm{AF}$, with special attention to the role of renal function.

\section{Materials and methods \\ Study population}

This is a single-center retrospective study aimed to assess the predictors of the long-term prognosis of AF catheter ablation. The present study enrolled $306 \mathrm{AF}$ patients who underwent first-time catheter ablation between January 2008 and April 2013 in the First Affiliated Hospital of Dalian Medical University. The inclusion criteria were as follows: age between 18 and 80 years, symptomatic AF despite the use of at least one antiarrhythmic drug, prior attempts of electrical cardioversion, and severe adverse events on rhythm-control drugs; exclusion criteria were defined as severe cardiac valvular diseases, left atrial diameter $(\mathrm{LAD})>50 \mathrm{~mm}$, left atrial thrombus, known bleeding diathesis, prior ablation for $\mathrm{AF}$, and other severe comorbidities resulting in intolerance of perioperative antiarrhythmic/anticoagulation drugs. As our design is a retrospective study, we therefore performed post-hoc power analyses through G"power software with parameters by default (effect size of 0.5 and $\alpha$ of 0.05 ). The estimated power for our study design was 0.99 , indicating our study has a high probability of detecting a real effect. All patients signed an informed written consent form to the study protocol that was approved by Ethics committee of First Affiliated Hospital of Dalian Medical University.

\section{Pre-procedure management}

For paroxysmal AF, low-molecular-weight heparin was administered in the pre-procedure period. For persistent AF, effective anticoagulation therapy with warfarin was performed targeting an international normalized ratio (INR) of 2 to 3 for more than 3 weeks. Warfarin was discontinued 5 days before the procedure and substituted with low-molecular-weight heparin Moreover, antiarrhythmic agents, except amiodarone, were discontinued for at least 5 half-lives before the procedure. Renal function was evaluated by the estimated glomerular filtration rate (eGFR) and creatinine clearance rate $(\mathrm{CCr})$
1-2 days before the procedure. eGFR was calculated by the abbreviated Modification of Diet in Renal Disease formula [7], and CCr was calculated by the Modification of the Cockcroft-Gault formula [8]. All patients underwent transthoracic echocardiography performed by two experienced senior cardiac sonographers. Left atrial volume (LAV) was assessed offline with Simpson's method using apical four-chamber and apical two-chamber views at ventricular end-systole [9] and indexed to body surface area calculated by the DuBois formula [10]. $\mathrm{CHADS}_{2}$ (congestive heart failure, hypertension, age $\geq 75$ years, type 2 diabetes, and previous stroke or transient ischaemic attack [doubled]) [11], $\mathrm{CHA}_{2} \mathrm{DS}_{2}$-VASc (congestive heart failure, hypertension, age $\geq 75$ years [doubled], type 2 diabetes, previous stroke, previous stroke or transient ischaemic attack [doubled], vascular disease, age 65 to 75 years, and sex category) [10] and $\mathrm{R}_{2} \mathrm{CHADS}_{2}$ (renal insufficiency [doubled], congestive heart failure, hypertension, age $\geq 75$ years, type 2 diabetes, and previous stroke or transient ischaemic attack [doubled]) [12] scores were calculated for each individual.

\section{Electrophysiological study and catheter ablation}

After exclusion of intracardiac thrombi by transesophageal echocardiography and assessment of pulmonary veins by $\mathrm{CT}$ scanning, catheter ablation was performed according to the HRS/EHRA/ECAS 2007 Consensus Statement on Catheter and Surgical Ablation of AF [13]. Circumferential pulmonary vein isolation $(\mathrm{CPVI})$ was performed for paroxysmal AF. and stepwise ablation was performed for persistent AF. If AF or atrial tachycardia continued despite the wide ablation above, pharmacolog$\mathrm{ical} /$ electrical cardioversion was performed afterwards.

\section{Post-procedure management}

After ablation, oral anticoagulation with warfarin was continued for at least 3 months, and subcutaneous lowmolecular-weight heparin injections were discontinued after targeting INR. Amiodarone was administered to persistent AF patients routinely with oral doses of $600 \mathrm{mg} /$ day for 1 week and $200 \mathrm{mg} /$ day for 3 months after ablation.

\section{Follow-up}

After discharge from the hospital, patients were followed up systematically at 1, 3, 6 and 12 months and then every 6 months in the outpatient department, including 24-h Holter recording and 12-lead electrocardiogram. Patients were encouraged to report palpitations and any other symptoms suggestive of tachycardia outside follow-up visits. Recurrence was defined as atrial tachyarrhythmias sustained for more than $30 \mathrm{~s}$ beyond a blanking period 
of 3 months [13], including AF, atrial flutter or atrial tachycardia.

\section{Statistical analyses}

Descriptive statistics are presented as the mean \pm standard deviation for continuous variables and as frequency (percentages) for categorical variables. Group comparisons were performed using the $\mathrm{t}$-test or $\mathrm{x}^{2}$-test, as appropriate. Multivariate logistic regression analysis was used to identify the associated factor for recurrence [15]. Receiver operating characteristic (ROC) curves were generated, and area under the curve (AUC) value was calculated to compare and evaluate the predictive performance of the independent predictors of AF recurrence after ablation. Survival analysis was performed using the R package "survival". Specifically, a Kaplan-Meier curve was generated for survival rates of patients with difference detection of log-rank test. Cox proportional hazards regression model was used to calculate hazard ratios (HRs) and 95\% confidence intervals (CIs) regarding recurrent-free survival (RFS). Specifically, we performed univariate Cox proportional hazards regression to identified RFSassociated clinical variables; variables significant in univariate analysis $(p<0.05)$ were involved in the multiple Cox proportional hazards regression model, which was further optimized through stepwise regression of backward elimination to detect independent prognostic predictors of recurrence of atrial tachyarrhythmia after catheter ablation for AF. For all statistical analysis, a two-tailed $p$-value less than 0.05 was considered statistically significant. Statistical analysis was conducted using SPSS 24.0, MedCalc 7.3 and R (version 4.0.2).

\section{Results \\ Baseline characteristics and procedure data}

A total of 306 patients were enrolled in the present study, including patients with persistent AF $(n=120)$ underwent stepwise ablation, and patients with paroxysmal AF $(\mathrm{n}=186)$ underwent CPVI (Fig. 1). The mean age was $56.7 \pm 10.4$ years. In total, 226 cases were male $(73.9 \%)$. There were 30 patients with CKD G1, 183 patients with CKD G2, 39 patients with CKD G3b, 53 patients with CKD G3a and 1 CKD G4 in our study. Patients included in this study were mainly mild to moderate renal insufficiency (CKD G2-CKD G3a). Following up $27.2 \pm 19.5$ months after a single procedure, 104 patients (34.0\%) experienced recurring atrial tachyarrhythmias (recurrence group), with $40.0 \%$ for persistent AF patients and $30.1 \%$ for paroxysmal AF patients. In addition, 202 patients maintained sinus rhythm without antiarrhythmic drugs (non-recurrence group). There were no significant differences in age, male sex, hypertension, diabetes mellitus, AF type, coronary heart disease, ischaemic stroke, $\mathrm{CHADS}_{2}$, $\mathrm{CHA}_{2} \mathrm{DS}_{2}$-VASc and $\mathrm{R}_{2} \mathrm{CHADS}_{2}$ scores, or procedure data (procedural time, $\mathrm{X}$-ray exposure time, ablation time) between the two groups (Table 1).

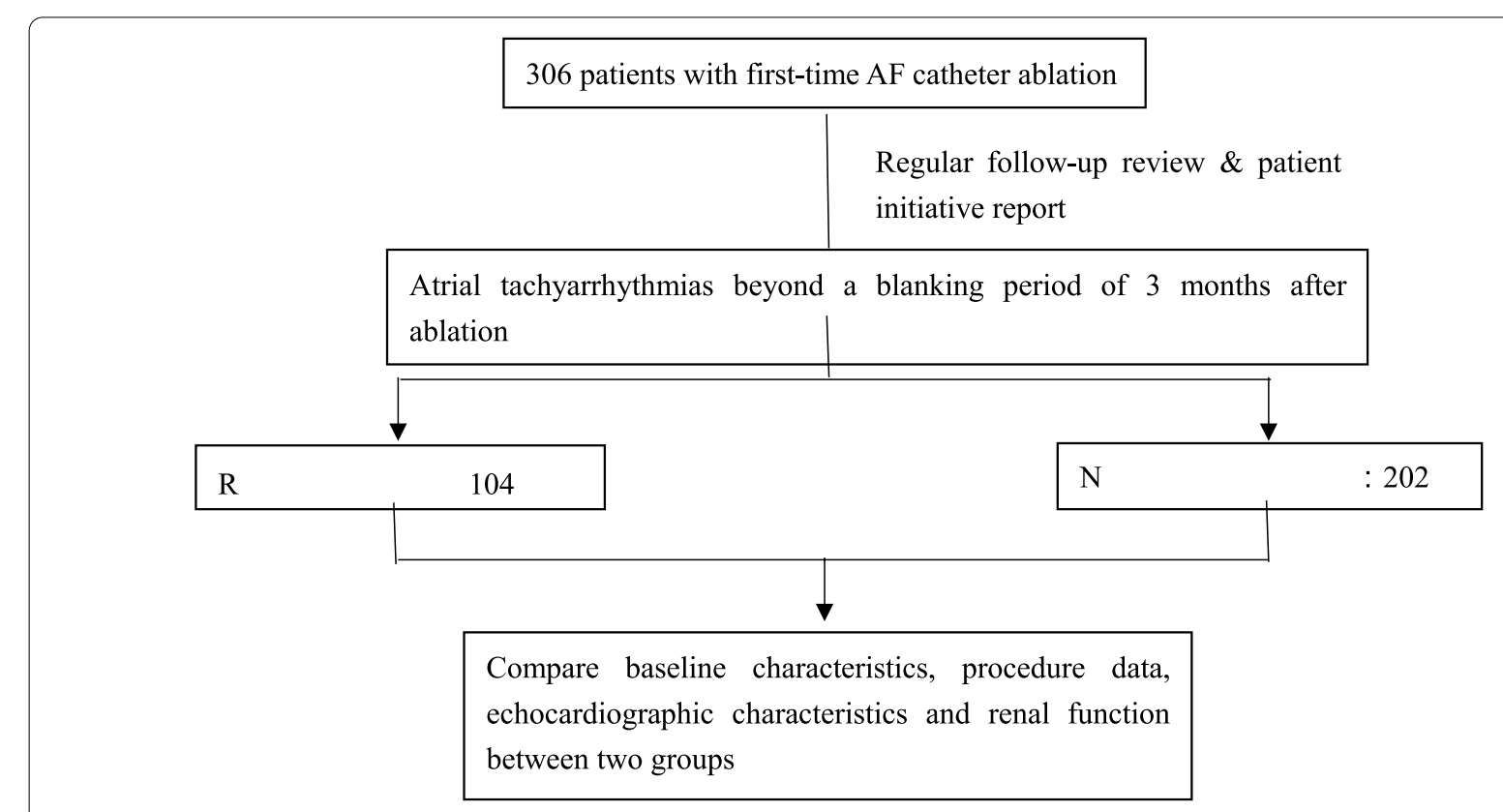

Fig. 1 Flowchart of the study. AF indicates atrial fibrillation 
Table 1 Baseline characteristics between recurrence and nonrecurrence groups

\begin{tabular}{|c|c|c|c|}
\hline & $\begin{array}{l}\text { Recurrence } \\
\text { Group }(n=104)\end{array}$ & $\begin{array}{l}\text { Non-recurrence } \\
\text { Group }(n=202)\end{array}$ & $P$ value \\
\hline Age, years & $57.0 \pm 10.9$ & $56.6 \pm 10.1$ & 0.464 \\
\hline Female, n (\%) & $31(29.8)$ & $49(24.3)$ & 0.295 \\
\hline History of AF, years & $3.0 \pm 1.5$ & $2.6 \pm 2.3$ & 0.848 \\
\hline Persistent AF, n (\%) & $48(46.2)$ & $72(35.6)$ & 0.065 \\
\hline Hypertension, n (\%) & $46(44.2)$ & $83(41.1)$ & 0.598 \\
\hline Diabetes mellitus, n (\%) & $6(5.8)$ & $23(11.4)$ & 0.112 \\
\hline $\begin{array}{l}\text { Coronary heart disease, } \\
\mathrm{n}(\%)\end{array}$ & $9(8.7)$ & $22(10.9)$ & 0.539 \\
\hline Ischaemic stroke, n (\%) & $8(7.7)$ & $12(5.9)$ & 0.557 \\
\hline $\mathrm{CHADS}_{2}$ score & $0.8 \pm 1.0$ & $0.8 \pm 0.9$ & 0.946 \\
\hline $\mathrm{CHA}_{2} \mathrm{DS}_{2}$-VASc score & $1.3 \pm 1.2$ & $1.2 \pm 1.2$ & 0.599 \\
\hline $\mathrm{R}_{2} \mathrm{CHADS}_{2}$ score & $1.6 \pm 1.4$ & $1.4 \pm 1.3$ & 0.082 \\
\hline Produce time, $\mathrm{h}$ & $2.5 \pm 1.5$ & $2.3 \pm 1.3$ & 0.265 \\
\hline Exposure time, $s$ & $61.6 \pm 29.3$ & $63.3 \pm 27.9$ & 0.176 \\
\hline Ablation time, min & $53.8 \pm 17.4$ & $55.6 \pm 19.9$ & 0.198 \\
\hline $\mathrm{LAD}, \mathrm{mm}$ & $41.6 \pm 5.0$ & $39.39 \pm 3.4$ & $<0.001$ \\
\hline$L A V, m l$ & $58.6 \pm 17.7$ & $50.5 \pm 11.6$ & $<0.001$ \\
\hline $\mathrm{LAVI}, \mathrm{ml} / \mathrm{m}^{2}$ & $33.7 \pm 9.7$ & $27.1 \pm 6.5$ & $<0.001$ \\
\hline LVEF, \% & $57.2 \pm 5.7$ & $59.9 \pm 4.8$ & 0.108 \\
\hline $\mathrm{eGFR}, \mathrm{ml} / \mathrm{min} / 1.73^{2}$ & $53.5 \pm 14.4$ & $65.5 \pm 13.3$ & $<0.001$ \\
\hline $\mathrm{CCr}, \mathrm{ml} / \mathrm{min}$ & $85.2 \pm 26.1$ & $101.5 \pm 29.4$ & 0.033 \\
\hline
\end{tabular}

\section{Echocardiographic characteristics}

Compared with the non-recurrence group, patients in the recurrence group had a higher LAD, LAV and LAVI (Table 1). Of note, multivariate logistic analysis suggested LAVI as an independent associated factor for long-term recurrence after AF ablation $(p<0.0001)$, outperforming either LAD $(p=0.034)$ or LAV $(p=0.598)$ (Table 2$)$. The ROC curve showed an AUC of 0.708 (95\% CI, 0.65 to $0.76, p<0.001$ ) for LAVI (Fig. 2). A cut-off point of $30 \mathrm{ml} /$ $\mathrm{m}^{2}$ of the LAVI had a specificity of $71.8 \%$ and a sensitivity of $62.5 \%$, with a positive/negative predictive value of $52.8 \% / 79.2 \%$ (Fig. 2, blue line). Increased LAVI contributed to higher recurrence (Fig. 3).

\section{Renal insufficiency}

The patients in the recurrence group had a lower eGFR $\left(53.5 \pm 14.4\right.$ vs. $\left.65.5 \pm 13.3 \mathrm{ml} / \mathrm{min} / 1.73^{2}, p<0.001\right)$ and $\mathrm{CCr}(85.2 \pm 26.1$ vs. $101.5 \pm 29.4 \mathrm{ml} / \mathrm{min}, p<0.05)$ than those in the non-recurrence group (Table 1). Multivariate logistic analysis indicated that eGFR was an independent associated factor for recurrence (Table 2). The AUC of eGFR was calculated to be 0.725 (95\% CI, 0.67 to $0.77, p<0.001$ ) (Fig. 2, yellow line). The optimal cutoff point for eGFR as an independent predictor was
Table 2 Multivariate logistic regression analysis of predictors for recurrence after AF ablation

\begin{tabular}{llll}
\hline Variable & B & Sig & $\mathbf{9 5 \% C l}$ \\
\hline Age & 0.45 & 0.361 & $0.950-1.152$ \\
Female & -0.815 & 0.314 & $0.936-1.169$ \\
History of AF & -0.700 & 0.065 & $0.236-1.045$ \\
Persistent AF & -0.841 & 0.218 & $0.113-1.645$ \\
Hypertension & -0.997 & 0.528 & $0.017-8.145$ \\
Diabetes mellitus & -1.962 & 0.292 & $0.224-5.401$ \\
Coronary heart disease & -2.026 & 0.281 & $0.003-5.265$ \\
Ischaemic stroke & -0.277 & 0.558 & $0.300-1.916$ \\
LAD & -0.360 & 0.034 & $0.504-0.967$ \\
LAV & -0.719 & 0.598 & $0.545-1.418$ \\
LAVI & -0.819 & 0.000 & $0.322-0.602$ \\
eGFR & -0.354 & 0.002 & $0.559-0.881$ \\
CCr & -0.007 & 0.637 & $0.966-1.021$ \\
\hline
\end{tabular}

$55 \mathrm{ml} / \mathrm{min} / 1.73^{2}$, with a specificity of $79.2 \%$ and a sensitivity of $51.9 \%$ and a positive/negative predictive value of $56.2 \% / 76.2 \%$ (Fig. 2). Patients with an eGFR $\leq 55 \mathrm{ml} /$ $\mathrm{min} / 1.73^{2}$ had a significantly increased rate of recurrence (Fig. 3).

\section{Independent prognostic value of eGFR and LAVI for recurrence prediction}

We then investigate the prognostic value of eGFR in evaluating recurrence of atrial tachyarrhythmia after catheter

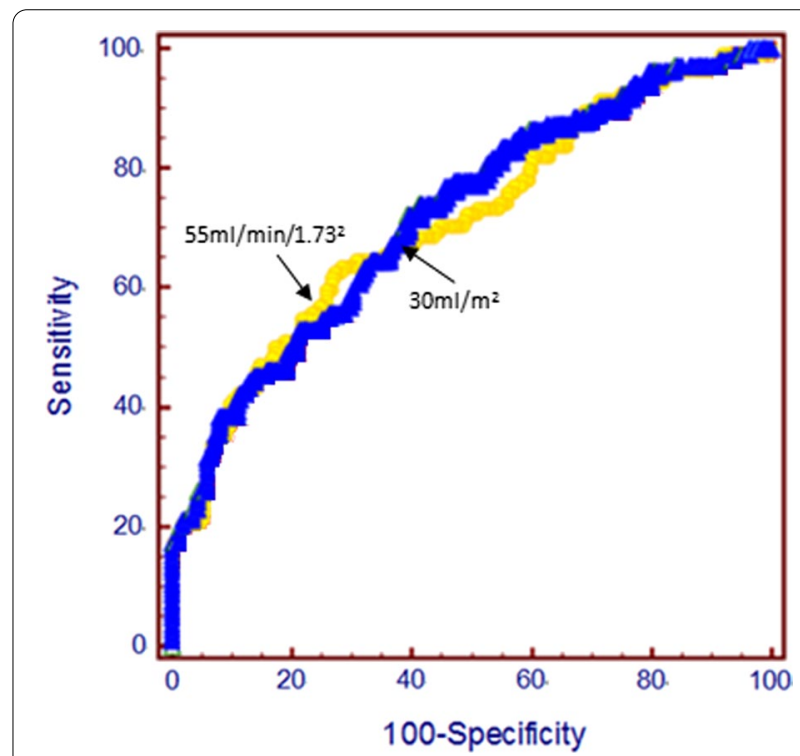

Fig. 2 The ROC curve analysis of the eGFR (yellow line) and LAVI (blue line) according to recurrence of AF after a single ablation procedure. Arrows indicate optimal cut-off point for sensitivity and specificity 


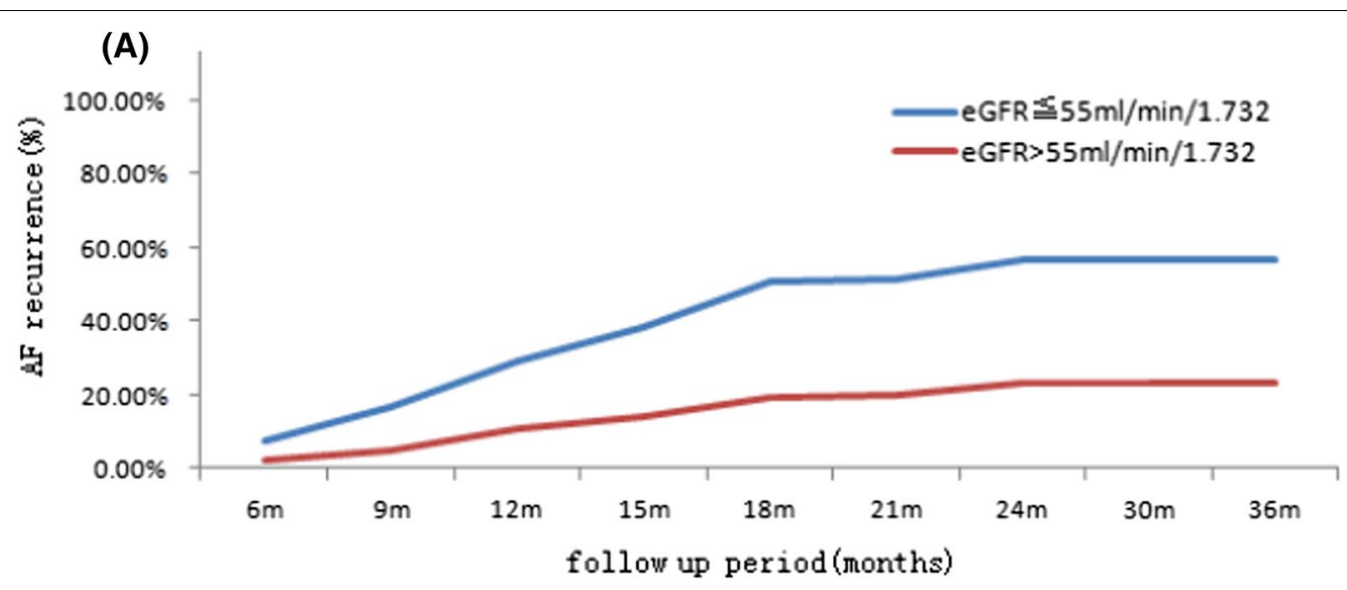

(B)

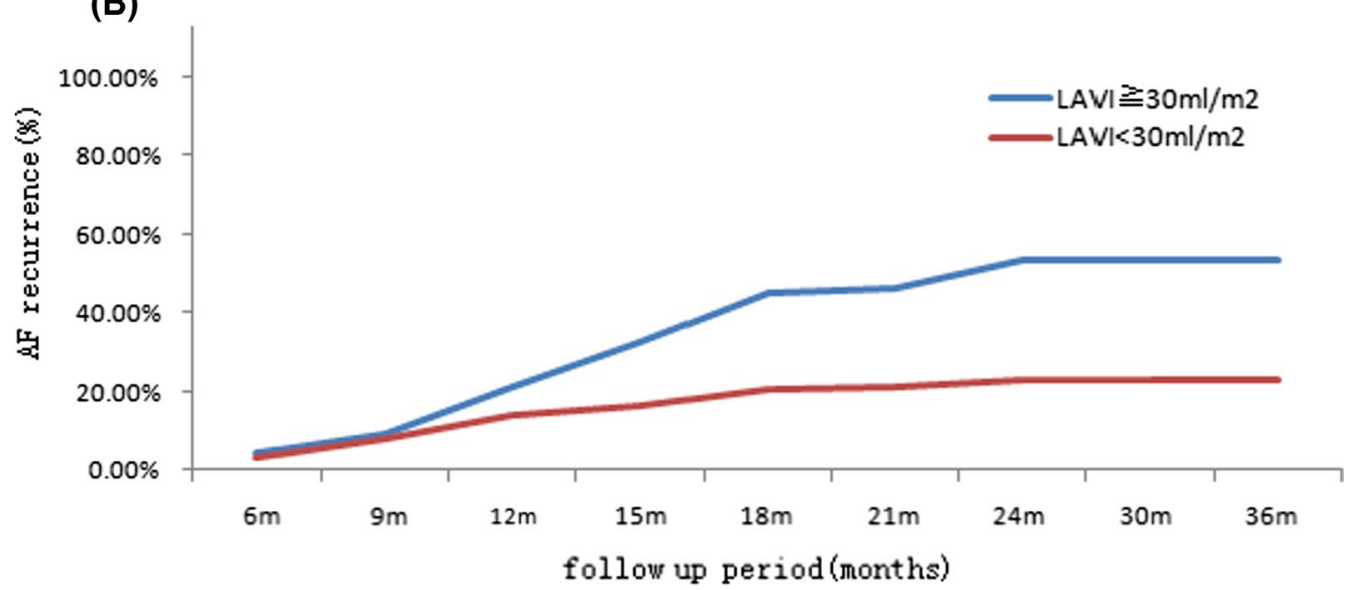

Fig. 3 Long-term AF recurrence after a single catheter ablation: A the long-term recurrence in patients with or without eGFR $\leq 55 \mathrm{ml} / \mathrm{min} / 1.73^{2}$. B The long-term recurrence in patients with or without $L A V I \geq 30 \mathrm{ml} / \mathrm{m}^{2}$

ablation for AF. In this manner, we considered time-toevent process and performed univariate Cox hazard proportional regression model for all clinical variables. We found history of AF (HR: 1.23, 95\% CI: 1.03-1.49, $p=0.025$ ), produce time (HR: $2.35,95 \%$ CI: $1.54-1.3 .58$, $p<0.001$ ), LAD (HR: 1.32 , 95\% CI: $1.21-1.44, p<0.001$ ), LAV (HR: $1.33,95 \%$ CI: $1.26-1.40, p<0.001$ ) and LAVI (HR: $1.32,95 \%$ CI: $1.25-1.40, p<0.001)$ presented with risk factors for recurrence; while CCr (HR: 0.94, 95\% CI: $0.92-0.95, p<0.001$ ), eGFR (HR: 0.96, 95\% CI: 0.940.97, $p<0.001$ ) and LVEF (HR: 0.5, 95\% CI: 0.92-0.98, $p=0.002)$ showed significant protective effect to recurrence (Table 3, Fig. 4).

For those variables reached statistical significance in univariate analysis, we constructed a multivariate Cox proportional hazard regression model with backward feature selection; such analysis identified four features, including eGFR (HR: 0.93, 95\% CI: 0.91-0.95, $p<0.001$ ), LAD (HR: $1.75,95 \%$ CI: 1.46-2.10, $p<0.001$ ), LAV (HR: 1.34, 95\% CI: $1.20-1.50, p<0.001)$ and LAVI (HR: 1.38 ,
95\% CI: $1.19-1.60, p<0.001)$, indicating that both eGFR and LAVI are independent prognostic factors for predicting recurrence of atrial tachyarrhythmia after catheter ablation for AF when adjusting other major clinical features (Table 3, Fig. 4). Using our pre-identified optimal cut-off, and patients with eGFR less or equal than $55 \mathrm{ml} /$ $\min / 1.73^{2}$ showed significant poor outcome than those with high eGFR level (log-rank test, $p=0.012$; Fig. 5a). Likewise, patients with higher LAVI $\left(>30 \mathrm{ml} / \mathrm{m}^{2}\right)$ presented unfavourable prognosis than those patients with lower LAVI level (log-rank test, $p<0.0001$; Fig. 5b).

\section{Discussion}

In the present study, a single ablation of AF was associated with a favourable outcome. Following up $27.2 \pm 19.5$ months, the overall recurrence rate was $34.0 \%$, with $40.0 \%$ for persistent AF patients and $30.1 \%$ for paroxysmal AF patients. Multivariate logistic regression analysis indicated that pre-procedural LAVI and eGFR were independent predictors of long-term 
Table 3 Univariate and multivariate Cox regression with backward elimination of clinical variables for predicting recurrence after AF ablation

\begin{tabular}{|c|c|c|c|c|}
\hline & Hazard ratio & Lower $95 \% \mathrm{Cl}$ & Upper $95 \% \mathrm{Cl}$ & $P$ value \\
\hline \multicolumn{5}{|l|}{ Univariate } \\
\hline Age, years & 1.004 & 0.983 & 1.026 & 0.718 \\
\hline \multicolumn{5}{|l|}{ Gender } \\
\hline \multicolumn{5}{|l|}{ Male (ref.) } \\
\hline Female & 1.258 & 0.821 & 1.926 & 0.291 \\
\hline \multicolumn{5}{|l|}{ Persistent AF } \\
\hline \multicolumn{5}{|l|}{ No (ref.) } \\
\hline Yes & 1.437 & 0.976 & 2.115 & 0.066 \\
\hline History of AF & 1.235 & 1.026 & 1.486 & 0.025 \\
\hline \multicolumn{5}{|l|}{ Hypertension } \\
\hline \multicolumn{5}{|l|}{ No (ref.) } \\
\hline Yes & 0.924 & 0.625 & 1.367 & 0.693 \\
\hline \multicolumn{5}{|l|}{ Diabetes } \\
\hline \multicolumn{5}{|l|}{ No (ref.) } \\
\hline Yes & 0.572 & 0.248 & 1.317 & 0.189 \\
\hline \multicolumn{5}{|l|}{ Coronary heart disease } \\
\hline \multicolumn{5}{|l|}{ No (ref.) } \\
\hline Yes & 0.778 & 0.389 & 1.554 & 0.477 \\
\hline \multicolumn{5}{|l|}{ Ischaemic stroke } \\
\hline \multicolumn{5}{|l|}{ No (ref.) } \\
\hline Yes & 1.336 & 0.648 & 2.756 & 0.433 \\
\hline $\mathrm{CCr}, \mathrm{ml} / \mathrm{min}$ & 0.935 & 0.920 & 0.951 & $<0.001$ \\
\hline $\mathrm{eGFR}, \mathrm{ml} / \mathrm{min} / 1.73^{2}$ & 0.955 & 0.941 & 0.970 & $<0.001$ \\
\hline $\mathrm{CHADS}_{2}$ score & 0.917 & 0.738 & 1.140 & 0.437 \\
\hline $\mathrm{CHA}_{2} \mathrm{DS}_{2}$-VASc score & 0.997 & 0.846 & 1.177 & 0.976 \\
\hline $\mathrm{R}_{2} \mathrm{CHADS}_{2}$ score & 0.996 & 0.959 & 1.035 & 0.849 \\
\hline Produce time, $\mathrm{h}$ & 2.350 & 1.542 & 3.582 & $<0.001$ \\
\hline Exposure time, $s$ & 0.997 & 0.981 & 1.013 & 0.706 \\
\hline Ablation time, min & 0.995 & 0.976 & 1.014 & 0.586 \\
\hline$L A D, m m$ & 1.319 & 1.209 & 1.439 & $<0.001$ \\
\hline LAV, ml & 1.327 & 1.257 & 1.401 & $<0.001$ \\
\hline LAVI, $\mathrm{ml} / \mathrm{m}^{2}$ & 1.323 & 1.251 & 1.400 & $<0.001$ \\
\hline LVEF, \% & 0.950 & 0.921 & 0.981 & 0.002 \\
\hline \multicolumn{5}{|c|}{ Multivariate (backward elimination) } \\
\hline $\mathrm{eGFR}, \mathrm{ml} / \mathrm{min} / 1.73^{2}$ & 0.930 & 0.912 & 0.947 & $<0.001$ \\
\hline $\mathrm{LAD}, \mathrm{mm}$ & 1.751 & 1.459 & 2.102 & $<0.001$ \\
\hline LAV, $\mathrm{ml}$ & 1.342 & 1.203 & 1.497 & $<0.001$ \\
\hline $\mathrm{LAVI}, \mathrm{ml} / \mathrm{m}^{2}$ & 1.379 & 1.186 & 1.602 & $<0.001$ \\
\hline
\end{tabular}

recurrence. A higher LAVI and a lower eGFR contributed to long-term recurrence after a single procedure for AF in mild to moderate renal insufficiency.

\section{$\mathrm{CHADS}_{2}, \mathrm{CHA}_{2} \mathrm{DS}_{2}-\mathrm{VASc}$, and $\mathrm{R}_{2} \mathrm{CHADS}_{2}$ score and AF}

Many factors have been proposed as predictors of prognosis after AF ablation, such as congestive heart failure, hypertension, diabetes, previous stroke and sex [14-17].
$\mathrm{CHADS}_{2}$ and $\mathrm{CHA}_{2} \mathrm{DS}_{2}$-VASc scores, widely used to predict stroke risks among patients with $\mathrm{AF}$, involve the abovementioned predictors. The $\mathrm{R}_{2} \mathrm{CHADS}_{2}$ score is a new stroke score that combines the $\mathrm{CHADS}_{2}$ score and an index of renal insufficiency. Chen et al. [18] demonstrated a positive relationship between new-onset AF incidence and $\mathrm{CHADS}_{2}$ scores in a prospective cohort study among Taiwanese patients. Further studies 


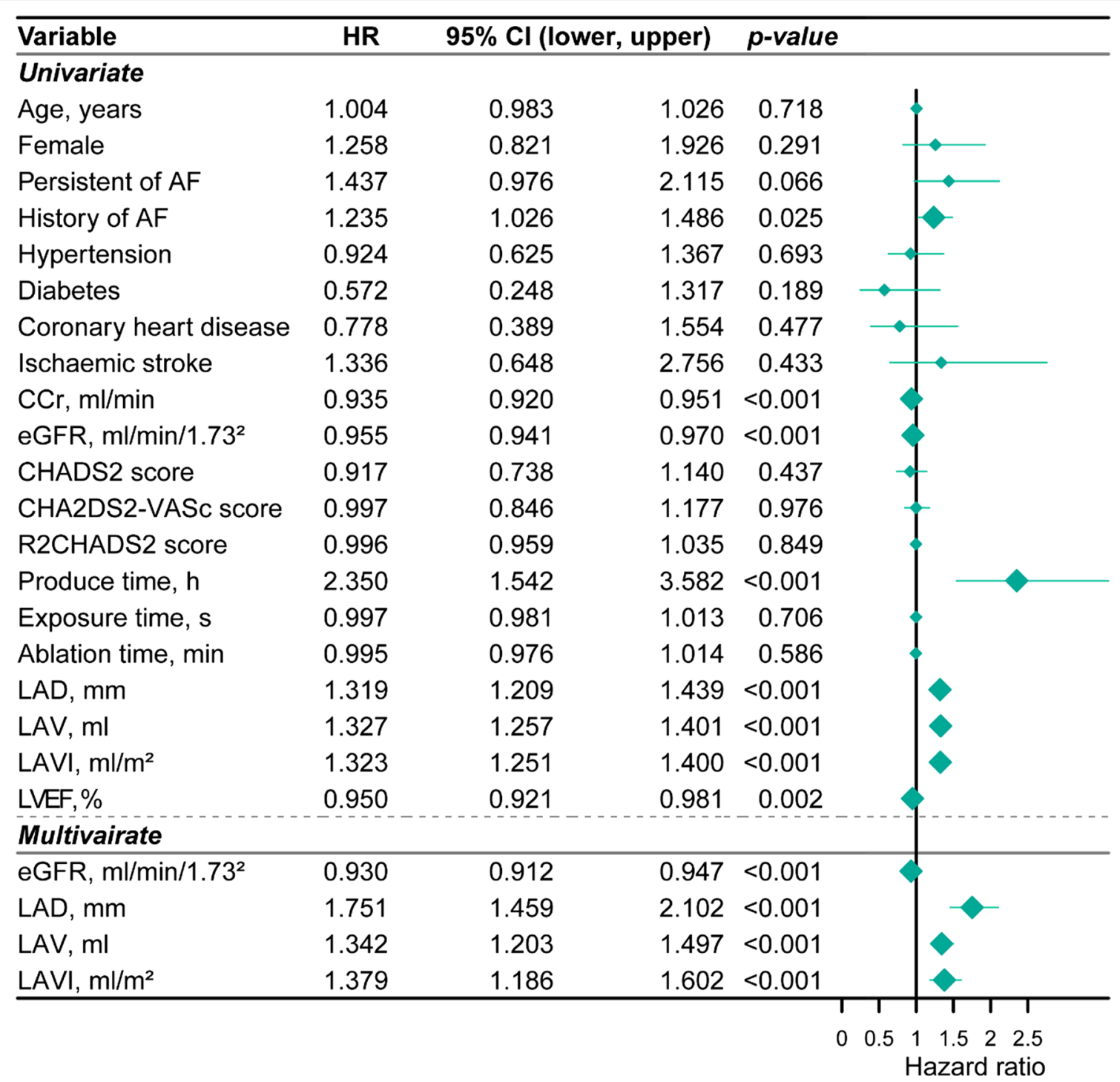

Fig. 4 Forestplot showing univariate and multivariate Cox proportional hazard regression results considering recurrence of atrial tachyarrhythmia after catheter ablation for AF by using clinical variables

identified a predictive value of $\mathrm{CHADS}_{2}, \mathrm{CHA}_{2} \mathrm{DS}_{2}$-VASc and $\mathrm{R}_{2} \mathrm{CHADS}_{2}$ scores for post-ablation recurrence of $\mathrm{AF}$ [18-20]. Letsas KP et al. [21] reported that the predictive accuracy of both $\mathrm{CHADS}_{2}$ and $\mathrm{CHA}_{2} \mathrm{DS}_{2}$-VASc was mediocre. Inconsistent with Kornej's and Chen's studies, the present study showed no significant differences in $\mathrm{CHADS}_{2}, \mathrm{CHA}_{2} \mathrm{DS}_{2}$-VASc, or $\mathrm{R}_{2} \mathrm{CHADS}_{2}$ scores between the recurrence and non-recurrence groups. This finding may be partly ascribed to a rigorous inclusion. Patients with severe comorbidities were excluded. In particular, most of the patients included in this retrospective analysis were mild to moderate renal insufficiency, which had no significant effect on $\mathrm{R}_{2} \mathrm{CHADS}_{2}$ score.

\section{Renal insufficiency and AF}

There is a higher prevalence of AF among patients with different stages of chronic renal disease [6]. $\mathrm{CCr}$ is widely used for the evaluation of renal failure in the clinic, while eGFR is preferred because it is more reliable, cheaper and easier to perform as a preoperative renal function test [22-24]. The $\mathrm{R}_{2} \mathrm{CHADS}_{2}$ score, involving an index of renal insufficiency determined as $\mathrm{CCr}$, may not effectively evaluate the effect of renal function on the prognosis of AF ablation. The REGARDS study further demonstrated that the prevalence of AF gradually increased with a decreasing eGFR [25]. In the present study, eGFR was calculated as a renal function index instead of $\mathrm{CCr}$, and 

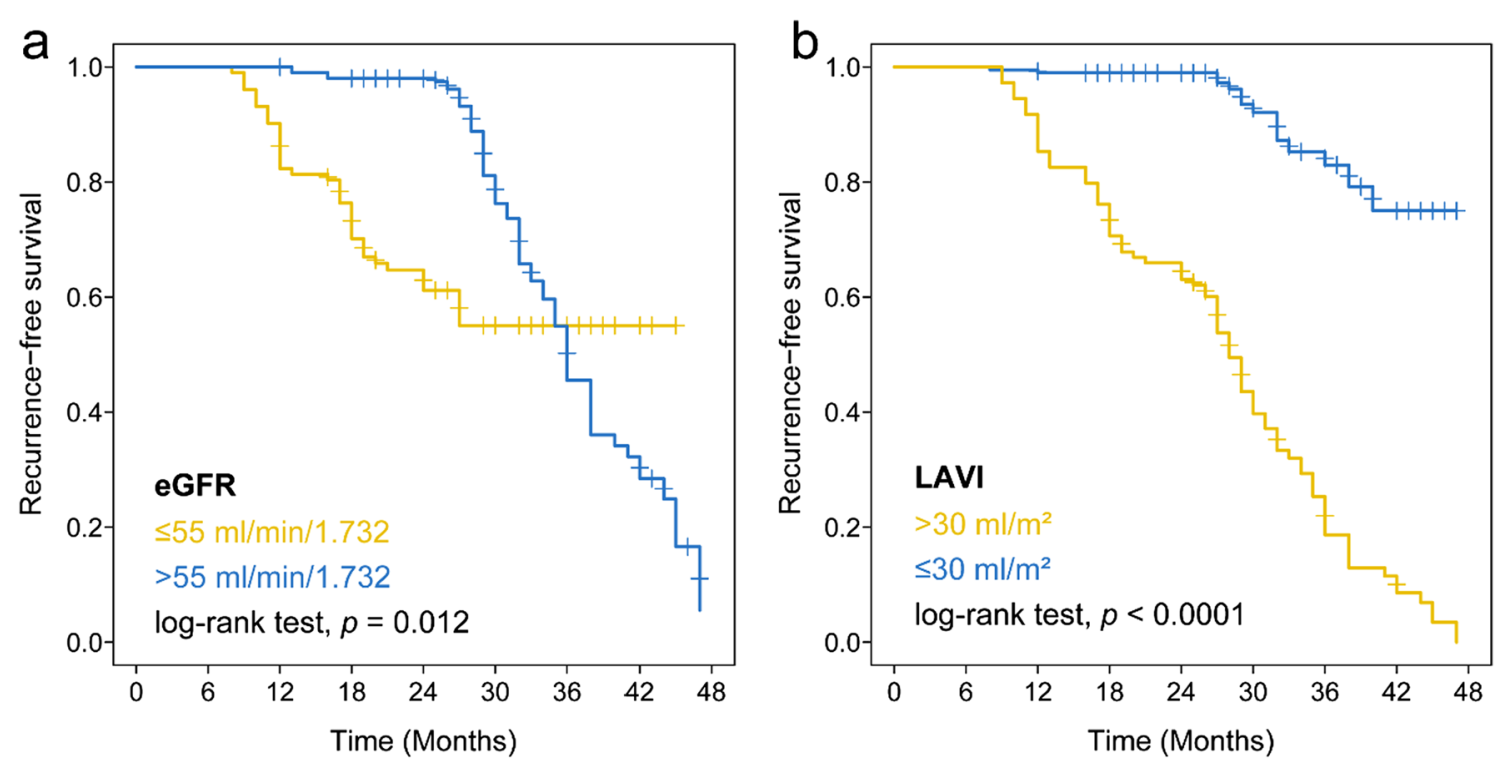

Fig. 5 Kaplan-Meier curve of recurrence-free survival between patients with different level of a eGFR $\left(\mathrm{ml} / \mathrm{min} / 1.73^{2}\right)$ and $\mathbf{b} L A V I\left(\mathrm{ml} / \mathrm{m}^{2}\right)$

multivariate logistic regression analysis showed that preprocedure eGFR was an independent associated factor of long-term recurrence. Furthermore, multivariate Cox proportional hazard regression model indicated eGFR as an independent prognostic factors for predicting recurrence. This confirmed that eGFR was superior to $\mathrm{CCr}$ as a prognostic index of AF ablation. Even mild renal insufficiency may have an unavoidable effect on recurrence after AF ablation.

Active sympathetic and renin-angiotensin-aldosterone systems (RAAS) play important roles in renal insufficiency [26-28], which also involves the pathogenesis of AF [29]. Norepinephrine released from sympathetic nerve endings enhances the $\mathrm{Ca}^{2+}$ transient, which may activate the $\mathrm{Na}^{+}-\mathrm{Ca}^{2+}$ exchange current and induce late phase 3 early afterdepolarization, resulting in focal discharge and AF [30, 31]. AngII and aldosterone were elevated in patients with renal insufficiency, and both could promote oxidative stress and atrial fibrosis, so-called atrial structural remodelling [32, 33]. Aldosterone also decreases the transient outward $\mathrm{K}^{+}$current and $\mathrm{I}_{\text {to }}$ density secondary to the rise in $\mathrm{Ca}^{2+}$ current, which generates abbreviation of action potential, the so-called atrial electrical remodelling, and induces AF [34]. Another workable mechanism for eGFR influencing on AF recurrence may be inflammation. Patients with renal insufficiency, even in the early stage, have been reported to have high expression of inflammatory factors, such as hypersensitive C-reactive protein, interleukin- 6 and fibrinogen $[35,36]$. In the early stage of renal insufficiency, inflammation could induce myocardial remodelling, which might result in recurrence of atrial arrhythmias after catheter ablation. Lin et al. [37] found that patients with higher hypersensitive $\mathrm{C}$-reactive protein levels had lower mean bipolar peak voltage in the LA, suggesting extensive atrial remodelling, severe substrate and a greater possibility of non-pulmonary vein triggers. Meanwhile, these patients have a relatively higher mean dominant frequency value and widely-distributed AF nests in the LA. In addition, $\mathrm{C}$-reactive protein may increase reactive oxygen species and enhance LA fibrosis, leading to atrial dilation and atrial dysfunction [38]. Therefore, the pathological mechanisms above may facilitate the recurrence of atrial tachyarrhythmia after AF ablation.

\section{Left atrial remodelling and $A F$}

Water-sodium retention, hyperactive sympathetic tone and RAAS activation induced by renal insufficiency [2628 ] increase LA volume overload and cause atrial remodelling. As reported in previously published studies, left atrial enlargement is the hallmark of atrial remodelling, which facilitates the prevalence of atrial arrhythmias, especially AF $[39,40]$. With the enlargement of the atria, progressive changes in cellular ultrastructure and extracellular matrix (composition and volume) develop. These abnormalities induce myocardial and interstitial fibrosis, local conduction heterogeneities and electrical dissociation between muscle bundles, consequently resulting in the initiation and perpetuation of $\operatorname{AF}[39,41]$. The persistence of atrial remodelling, potentially explaining arrhythmogenic substrates, is incremental to the postprocedure recurrence of AF [42, 43]. 
Hui-Ling Lee et al. [44] confirmed a larger LAD was demonstrated to increase the probability of AF recurrence after surgery significantly by a three-year longitudinal study. Despite its procurability, its validity has recently been challenged, as the LA is an asymmetrical cavity. Conversely, biplane LAV provides an overall and reproducible estimation of left atrial size when compared with reference standards such as magnetic resonance imaging. Considering the individual differences, LAVI, calculated as LAV indexed to body surface area, is more comparable in accuracy and reproducibility. Marchese et al. [45] proved that LAVI was a more exact estimate of LA remodelling than LAD. LAVI was strongly associated with the risk of AF recurrence after cardioversion, with a cut-off of $31 \mathrm{ml} / \mathrm{m}^{2}$. Kataoka et al. [46] demonstrated LAVI in predicting failure of the surgical maze procedure for AF patients. However, the role of LAVI in the prognosis of AF ablation has not been well-understood.

In the present study, we found that LAD, LAV and LAVI were higher in the recurrence group. Notably, multivariate logistic analysis demonstrated that LAVI, outperforming either LAV or LAD, was an independent associated factor for predicting long-term recurrence status after catheter ablation of AF. Specifically, increased LAVI contributed to long-term recurrence, with an optimal cut-off of $30 \mathrm{ml} / \mathrm{m}^{2}$, indicating that LAVI, characterized as left atrial remodelling, may be an important determinant for the prognosis of AF ablation. Additionally, multivariate Cox proportional hazard regression also indicated that both eGFR and LAVI are independent prognostic factor for AF recurrence after a single procedure.

Recurrence after AF ablation has remained a puzzle for both doctors and patients. Evaluation of the risk factors for recurrence is crucial in boosting the success rate. In this manner, we found that a decreased eGFR and an increased LAVI had significant adverse effects in predicting long-term recurrence status after a single procedure. Therefore, the preprocedural eGFR and LAVI might be taken into consideration for optimal patient enrolment for AF ablation. Furthermore, patients with $\mathrm{eGFR} \leq 55 \mathrm{ml} / \mathrm{min} / 1.73^{2}$ or $\mathrm{LAVI} \geq 30 \mathrm{ml} /$ $\mathrm{m}^{2}$ may be more challenging to maintain sinus rhythm after cardioversion, and require more aggressive clinical interventions.

We acknowledged several limitations. Firstly, patients involved in this study were mainly mild to moderate renal insufficiency (CKD G2-CKD G3a) with mean eGFR $61.4 \pm 14.8 \mathrm{ml} / \mathrm{min} / 1.73^{2}$, and these patients with severe renal insufficiency were neglected. The current data may not sufficient to fully assess renal function in the prognosis of AF ablation. According to clinical practice, patients with severe renal insufficiency (CKD G4-5) are mostly complicated with multiple diseases, and most of them are willing to accept conservative drug-treatment. Nevertheless, increasing numbers of patients with severe renal insufficiency are likely to choose AF ablation. It is expected that the sample size will be expanded in the future to evaluate the success rate and postoperative recurrence rate of $\mathrm{AF}$ ablation in patients with $\mathrm{CKD}$ G4-5, so as to provide reference for the selection of AF treatment options in these patients. Secondly, this study is a retrospective study, all patients were treated with standard surgery, but there were still differences in surgical details among different operators, which might affect the prognosis. In addition, the collected data might be not comprehensive enough, and other important influencing factors, even those related to renal function, may also be omitted. Thirdly, there was a lack of laboratory indicators for further study of the mechanism, such as inflammatory markers, LA fibrosis and LA voltage. Fourthly, AF recurrence rates may be underestimated by ignoring asymptomatic paroxysmal AF due to the limited follow-up period. Mobile health technology may improve the comprehensive management of AF [47]. Further investigation is needed to determine whether improving renal insufficiency can enhance the long-term success of catheter ablation for AF.

\section{Conclusions}

The present study showed that decreased eGFR and elevated LAVI contribute to increased recurrence after AF ablation. Renal insufficiency and LA remodelling might be important determinants for the long-term prognosis of AF ablation.

\section{Abbreviations}

AF: Atrial fibrillation; LA: Left atrium; LAD: Left atrial diameter; LAV: Left atrial volume; LAVI: Left atrial volume index; INR: International normalized ratio; eGFR: Estimated glomerular filtration rate; CCr: Creatinine clearance rate; CPVI: Circumferential pulmonary vein isolation; ROC: Receiver operating characteristic; AUC: Area under the curve; RAAS: Renin-angiotensin-aldosterone systems.

\section{Acknowledgements}

This work was funded by Guiding Scientific and Technological Project of Quzhou (2019063) to JZ.

\section{Authors' contributions}

$Z G, Y D$ and $Y X$ guided the design of conceptualization and methodology, furthermore ZG was responsibility for the research activity planning and execution. JZ wrote the original manuscript text. JZ, DZ and KC finished the data collation. JZ and DZ prepared Tables 1, 2 and 3. JZ and KC prepared

Figs. 1, 2, 3, 4 and 5. ZG and YD were responsible for manuscript review and editing. All authors reviewed the manuscript. All authors read and approved the final manuscript.

\section{Funding}

None 


\section{Availability of data and materials}

The datasets used and/or analysed during the current study are available from the corresponding author on reasonable request.

\section{Declarations}

\section{Ethics approval and consent to participate}

This study protocol for involving human data was in accordance with the Declaration of Helsinki, and was approved by Ethics committee of First Affiliated Hospital of Dalian Medical University (Ethics Reference NO: YJ-KY-FB-2013-50). Design and implementation of this study followed the HRS/EHRA/ECAS 2007 Consensus Statement on Catheter and Surgical Ablation of AF (http://doi.org/ 10.1016/j.hrthm.2007.04.005), including indications, surgical procedures, and drug treatment regimens. All patients signed an informed written consent form.

\section{Consent for publication}

Written informed consent for publication was obtained from all participants.

\section{Competing interests}

The authors declare that they have no competing interests.

Received: 25 May 2021 Accepted: 6 October 2021

Published online: 21 October 2021

\section{References}

1. Joseph PG, Healey JS, Raina P, et al. Global variations in the prevalence, treatment, and impact of atrial fibrillation in a multi-national cohort of 153, 152 middle-aged individuals. Cardiovasc Res. 2020;117:cvaa241.

2. Vinter $N$, Huang $Q$, Fenger-Grøn $M$, et al. Trends in excess mortality associated with atrial fibrillation over 45 years (Framingham Heart Study): community based cohort study. BMJ. 2020;370:m2724.

3. Lee KJ, Kim BJ, Han MK, et al. Effect of heart rate on stroke recurrence and mortality in acute ischemic stroke with atrial fibrillation. Stroke. 2020;51(1):162-9.

4. Cappato R, Calkins H, Chen SA, et al. Updated worldwide survey on the methods, efficacy, and safety of catheter ablation for human atrial fibrillation. Circ Arrhythm Electrophysiol. 2010;3(1):32-8.

5. Poole JE, Bahnson TD, Monahan KH, et al. Recurrence of atrial fibrillation after catheter ablation or antiarrhythmic drug therapy in the CABANA trial. J Am Coll Cardiol. 2020;75(25):3105-18.

6. Guo Y, Gao J, Ye P, et al. Comparison of atrial fibrillation in CKD and nonCKD populations: a cross-sectional analysis from the Kailuan study. Int J Cardiol. 2019;15(277):125-9.

7. Levey AS, Bosch JP, Lewis JB, et al. A more accurate method to estimate glomerular filtration rate from serum creatinine: a new prediction equation. Modification of diet in renal disease study group. Ann Intern Med. 1999;130:461-70.

8. Cockcroft DW, Gault MH. Prediction of creatinine clearance from serum creatinine. Nephron. 1976;16:31-41.

9. Lang RM, Bierig M, Devereux RB, et al. American Society of Echocardiography's Nomenclature and Standards Committee; Task Force on Chamber Quantification; American College of Cardiology Echocardiography Committee; American Heart Association; European Association of Echocardiography, European Society of Cardiology: recommendations for chamber quantification. Eur J Echocardiogr. 2006;7:79-108.

10. Du Bois D. A formula to estimate the approximate surface area if height and weight be known. Nutrition. 1989;5:303-11.

11. Chao TF, Lin YJ, Tsao HM, et al. CHADS(2) and CHA(2)DS(2)-VASc scores in the prediction of clinical outcomes in patients with atrial fibrillation after catheter ablation. J Am Coll Cardiol. 2011;58:2380-5.

12. Piccini JP, Stevens SR, Chang Y, et al. ROCKET AF Steering Committee and Investigators: Renal dysfunction as a predictor of stroke and systemic embolism in patients with nonvalvular atrial fibrillation: validation of the R(2)CHADS(2) index in the ROCKET AF (Rivaroxaban Once-daily, oral, direct factor $\mathrm{X}$ a inhibition Compared with vitamin $\mathrm{K}$ antagonism for prevention of stroke and Embolism Trial in Atrial Fibrillation) and ATRIA
(AnTicoagulation and Risk factors In Atrial fibrillation) study cohorts. Circulation. 2013;127:224-32.

13. Calkins H, Brugada J, Packer DL, Cappato R, Chen SA, Crijns HJ, Damiano RJ Jr, Davies DW, Haines DE, Haissaguerre M, lesaka Y. HRS/EHRA/ ECAS expert Consensus Statement on catheter and surgical ablation of atrial fibrillation: recommendations for personnel, policy, procedures and follow-up. A report of the Heart Rhythm Society (HRS) Task Force on catheter and surgical ablation of atrial fibrillation. Heart Rhythm. 2007;4(6):816-61.

14. Caldentey G, Khairy P, Roy D, et al. Prognostic value of the physical examination in patients with heart failure and atrial fibrillation: insights from the AF-CHF Trial (Atrial Fibrillation and Chronic Heart Failure). JACC Heart Fail. 2014;2:15-23.

15. Thacker EL, McKnight B, Psaty BM, et al. Association of body mass index, diabetes, hypertension, and blood pressure levels with risk of permanent atrial fibrillation. J Gen Intern Med. 2013;28:247-53.

16. Voskoboinik $A$, et al. Alcohol abstinence in drinkers with atrial fibrillation. N Engl J Med. 2020;382(1):20-8

17. Cove $C L$, Albert CM, Andreotti F, et al. Female sex as an independent risk factor for stroke in atrial fibrillation: possible mechanisms. Thromb Haemost. 2014;111:385-91.

18. Chao TF, Liu CJ, Chen SJ, et al. CHADS2 score and risk of new-onset atrial fibrillation: a nationwide cohort study in Taiwan. Int J Cardiol. 2013;168:1360-3.

19. Bai Y, Liu N, Bai R, Wu JH, et al. Impacts of radiofrequency ablation on quality of life of atrial fibrillation patients with low CHA2DS2-VASC score. Zhonghua Nei Ke Za Zhi. 2016;55(4):278-82.

20. Kornej J, Hindricks G, Kosiuk J, et al. Comparison of CHADS2, R2CHADS2, and CHA2DS2-VASc scores for the prediction of rhythm outcomes after catheter ablation of atrial fibrillation: the Leipzig Heart Center AF Ablation Registry. Circ Arrhythm Electrophysiol. 2014;7:281-7.

21. Letsas KP, Efremidis M, Giannopoulos G, et al. CHADS2 and CHA2DS2VASc scores as predictors of left atrial ablation outcomes for paroxysmal atrial fibrillation. Europace. 2014;16:202-7.

22. Inker LA, Astor BC, Fox CH, et al. KDOQI US commentary on the 2012 KDIGO clinical practice guideline for the evaluation and management of CKD. Am J Kidney Dis. 2014;63:713-35.

23. Iwasaki Y, Sawada T, Kijima H, et al. Estimated glomerular filtration rate is superior to measured creatinine clearance for predicting postoperative renal dysfunction in patients undergoing pancreatoduodenectomy. Pancreas. 2010;39:20-5.

24. Fiseha T, Mengesha T, et al. Estimation of renal function in adult outpatients with normal serum creatinine. BMC Res Notes. 2019;12(1):462.

25. Baber U, Howard VJ, Halperin JL, et al. Association of chronic kidney disease with atrial fibrillation among adults in the United States REasons for Geographic and Racial Differences in Stroke (REGARDS) study. Circ Arrhythm Electrophysiol. 2011;4:26-32.

26. Meyer C, Schueller P, Balzer J, et al. Sympathetic hyperactivity influences chemosensor function in patients with end-stage renal disease. Eur J Med Res. 2009;14:151-5.

27. Kumar U, Wettersten N, Garimella PS. Cardiorenal syndrome: pathophysiology. Cardiol Clin. 2019;37(3):251-65.

28. McCullough PA, Kellum JA, Haase M, et al. Pathophysiology of the cardiorenal syndromes: executive summary from the eleventh consensus conference of the Acute Dialysis Quality Initiative (ADQI). Contrib Nephrol. 2013;182:82-98.

29. Potpara ST, Jokic V, Dagres N, Marin F, Prostran SM, et al. Cardiac arrhythmias in patients with chronic kidney disease: implications of renal failure for antiarrhythmic drug therapy. Curr Med Chem. 2016;23(19):2070-83.

30. Patterson E, Lazzara R, Szabo B, et al. Sodium-calcium exchange initiated by the Ca2+ transient: an arrhythmiatrigger within pulmonary veins. J Am Coll Cardiol. 2006;47:1196-206.

31. Oral H, Crawford T, Frederick M, et al. Inducibility of paroxysmal atrial fibrillation by isoproterenol and its relation to the mode of onset of atrial fibrillation. J Cardiovasc Electrophysiol. 2008;19:466-70.

32. Tsai CF, Yang SF, Chu HJ, Ueng KC. Cross-talk between mineralocorticoid receptor/angiotensin II type 1 receptor and mitogen-activated protein kinase pathways underlies aldosterone-induced atrial fibrotic responses in HL-1 cardiomyocytes. Int J Cardiol. 2013;169:17-28. 
33. Mayyas F, Alzoubi KH, Van Wagoner DR. Impact of aldosterone antagonists on the substrate for atrial fibrillation: aldosterone promotes oxidative stress and atrial structural/electrical remodelling. Int J Cardiol. 2013;168:5135-42.

34. Perrier R, Richard S, Sainte-Marie Y, et al. A direct relationship between plasma aldosterone and cardiac L-type $\mathrm{Ca}^{2+}$ current in mice. J Physiol. 2005;569:153-62.

35. Pan J, Wang W, Wu X, et al. Inflammatory cytokines in cardiac pacing patients with atrial fibrillation and asymptomatic atrial fibrillation. Panminerva Med. 2018;60(3):86-91.

36. Macisaac RJ, Ekinci El, Jerums G. Markers of and risk factors for the development and progression of diabetic kidney disease. Am J Kidney Dis. 2014;63(2):S39-62.

37. Lim HS, Schultz C, Dang J, et al. Time course of inflammation, myocardia injury, and prothrombotic response after radiofrequency catheter ablation for atrial fibrillation. Circ Arrhythm Electrophysiol. 2014;7:83-9.

38. Toyama K, Yamabe H, Uemura T, et al. Analysis of oxidative stress expressed by urinary level of 8-hydroxy-2'-deoxyguanosine and biopyrrin in atrial fibrillation: effect of sinus rhythm restoration. Int J Cardiol. 2013;168:80-5

39. Teh AW, Kistler PM, Lee G, et al. Electroanatomic remodeling of the left atrium in paroxysmal and persistent atrial fibrillation patients without structural heart disease. J Cardiovasc Electrophysiol. 2012;23:232-8.

40. Akkaya M, Higuchi K, Koopmann M, et al. Higher degree of left atrial structural remodeling in patients with atrial fibrillation and left ventricular systolic dysfunction. J Cardiovasc Electrophysiol. 2013;24:485-91.

41. Guichard JB, Naud P, Xiong F, et al. Comparison of atrial remodeling caused by sustained atrial flutter versus atrial fibrillation. JACC. 2020;76(4):374-88.
42. Park J, Joung B, Uhm JS, et al. High left atrial pressures are associated with advanced electroanatomical remodeling of left atrium and independent predictors for clinical recurrence of atrial fibrillation after catheter ablation. Heart Rhythm. 2014;11:953-60.

43. Ejima K, Kato K, Arai K, et al. Impact of atrial remodeling on the outcome of radiofrequency catheter ablation of paroxysmal atrial fibrillation. Circ J. 2014;78:872-7.

44. Lee H-L, Hwang Y-T, Chang P-C, et al. A three-year longitudinal study of the relation between left atrial diameter remodeling and atrial fibrillation ablation outcome. J Geriatr Cardiol. 2018;15(7):486-91.

45. Marchese $P$, Malavasi $\bigvee$, Rossi L, et al. Indexed left atrial volume is superior to left atrial diameter in predicting nonvalvular atrial fibrillation recurrence after successful cardioversion: a prospective study. Echocardiography. 2012;29:276-84.

46. Kataoka T, Hamasaki S, Inoue K, et al. Left atrium volume index and pathological features of left atrial appendage as a predictor of failure in postoperative sinus conversion. J Cardiol. 2010;55:274-82.

47. Guo Y, Lane DA, Wang $L$, et al. Mobile health technology to improve care for patients with atrial fibrillation. J Am Coll Cardiol. 2020;75(13):1523-34.

\section{Publisher's Note}

Springer Nature remains neutral with regard to jurisdictional claims in published maps and institutional affiliations.
Ready to submit your research? Choose BMC and benefit from:

- fast, convenient online submission

- thorough peer review by experienced researchers in your field

- rapid publication on acceptance

- support for research data, including large and complex data types

- gold Open Access which fosters wider collaboration and increased citations

- maximum visibility for your research: over 100M website views per year

At BMC, research is always in progress.

Learn more biomedcentral.com/submissions 